\title{
Costo Oportunidad de Adolescentes en Unidades de Medicina Familiar
}

\author{
PATRICIA UGALDE V. ${ }^{1,2}$, ENRIQUE VILLARREAL R. ${ }^{1}$, EMMA ROSA VARGAS D. ${ }^{1}$, \\ LIDIA MARTÍNEZ G. ${ }^{1}$, LILIANA GALICIA R. ${ }^{1}$ \\ 1. Doctor. Unidad de Investigación Epidemiológica y en Servicios de Salud Querétaro. Instituto Mexicano del Seguro Social. \\ Querétaro, México. \\ 2. Licenciado. Unidad de Medicina Familiar No. 16 "Dr. Arturo Guerrero Ortiz" Querétaro. Instituto Mexicano del Seguro \\ Social. Querétaro, México.
}

\begin{abstract}
Opportunity Cost for Adolescents at Family Medicine Clinics

Objective: To determine the opportunity cost for adolescents requiring care at Family Medicine Clinics. Material and Methods: Study of cost performed on 624 patients, 10-19 years of age, Family Medicine Clinics, Pharmacy and Laboratory. A conglomerate sample technique was used (Medical Unit), and proportional sampling internally. Several sociodemographic variables were evaluated, including transfer, waiting time and care. Statistical evaluation included averages, percentages, and confidence intervals of 95\%. Results: The cost-opportunity for Family Medicine and Pharmacy in the situations evaluated was $\$ 10.22$ for the teenager, $\$ 71.43$ for the first family member, and $\$ 14.28$ for the second person accompanying the child. When they attend all three services, the cost is $\$ 12.26, \$ 82.71$ and $\$ 16.54$ respectively. A weighted average cost of opportunity IFOR all three services is $\$ 93.18$. Conclusions: The largest cost of opportunity for adolescents occurs when waiting for care in Family Medicine. It is suggested that strategies be implemented to decrese waiting times in the various services.
\end{abstract}

(Key words: Family Medicine Clinics, adolescent, cost-opportunity).

Rev Chil Pediatr 2010; 81 (5): 418-424

\section{RESUMEN}

Objetivo. Determinar el costo oportunidad de los adolescentes que demandan atención en las Unidades de Medicina Familiar. Material y Métodos. Estudio de costo oportunidad realizado en 624 usuarios de 10 a 19 años de edad atendidos en tres Unidades de Medicina Familiar, en los servicios de Medicina Familiar, Farmacia y Laboratorio. Se empleó la técnica muestral por conglomerados (Unidad Médica) y al interior por cuota. Se estudiaron variables sociodemográficas y número de acompañantes; se estimó el costo oportunidad para

Trabajo recibido el 24 de febrero de 2010, devuelto para corregir el 06 de julio de 2010, segunda versión el 19 de julio de 2010 , tercera versión el 15 de agosto de 2010, aceptado para publicación el 07 de septiembre de 2010.

Correspondencia a:

Enrique Villarreal R.

E-mail: felibree@infosel.net.mx 
el traslado, espera y atención. El análisis estadístico incluyó promedios, porcentajes e intervalos de confianza del 95\%. Resultados: El costo oportunidad promedio para Medicina Familiar y Farmacia es de \$10.22 para el adolescente, de $\$ 71.43$ para el acompañante 1 y de $\$ 14.28$ para el acompañante 2; cuando acuden a los tres servicios es de $\$ 12.26$, $\$ 82.71$ y $\$ 16.54$ respectivamente. El costo oportunidad promedio ponderado cuando acuden a los tres servicios es de $\$ 93.18$. Conclusión: El mayor costo oportunidad para los adolescentes corresponde a la espera en el servicio de Medicina Familiar. Por lo que se sugiere implementar estrategias que disminuyan el tiempo de espera en los diferentes servicios.

(Palabras clave: Costo-oportunidad, adolescentes, medicina familiar).

Rev Chil Pediatr 2010; 81 (5): 418-424

\section{Introducción}

La demanda de servicios de salud aumenta cada día ${ }^{1}$. Debido a las limitaciones de recursos económicos, y el alto costo que representan para la sociedad los servicios de salud, se han introducido herramientas de evaluación económica aplicadas a la salud, con la finalidad de evaluar la efectividad y eficacia de las nuevas tecnologías y servicios ${ }^{2,3}$. La economía de la salud es la ciencia que se encarga del estudio de la asignación de recursos dentro de este sector con el objetivo de maximizar el bienestar social en términos de salud ${ }^{4}$. El impacto de las intervenciones sanitarias sobre los recursos consumidos se evalúa a través de análisis que incluyen: costo-efectividad, costobeneficio, costo-utilidad y costo-oportunidad ${ }^{5}$. El costo-oportunidad, se define como el costo de la mejor alternativa desechada. Expresa el verdadero costo de una inversión, la oportunidad que se ha perdido de usar los recursos disponibles en otra necesidad, debido a que se considera, se están utilizando en la mejor opción $^{3,6}$ y el tiempo es el indicador más usado para evaluarlo. El costo del tiempo se estima a través de la actividad remunerada o no remunerada. En el área de la salud, el costo del tiempo se aplica en el traslado, espera y atención de los diferentes servicios de atención médica ${ }^{5,7,8}$. Existen pocos estudios que evalúan el costo-oportunidad de los adultos ${ }^{9-11}$ y niños ${ }^{12}$ que acuden a las unidades de medicina familiar, pero no existen estudios reportados sobre el costo-oportunidad en adolescentes quienes representan un grupo etario importante, en países en desarrollo existen más de 1000 millones ${ }^{13}$. En Chile, este grupo corresponde al $22 \%$ de la población total ${ }^{14}$. En México representa el $21 \%$ de la pirámide poblacional y $14,1 \%$ de la población adscrita a Médico familiar, con un perfil de uso anual de 2,68 consultas ${ }^{15,16}$. Los adolescentes conforman un grupo de especial interés para la salud pública por ser un blanco indispensable de los programas preventivos ${ }^{17}$. Por lo que, el objetivo de esta investigación es determinar el costo-oportunidad de los adolescentes que demandan atención en las Unidades de Medicina Familiar.

\section{Pacientes y Métodos}

Estudio de costo oportunidad en adolescentes (10 a 19 años) usuarios de los servicios de Medicina Familiar, Farmacia y Laboratorio de las Unidades de Medicina Familiar número 9, 13 y 16 del Instituto Mexicano del Seguro Social, Delegación Querétaro, de enero a febrero de 2010. Se excluyeron aquellos que se retiraron sin haber recibido atención, que fueron referidos al servicio de Atención Médica Continua o al servicio de Urgencias. La muestra (624) se obtuvo mediante la fórmula de promedios para población infinita $(\mathrm{n}=$ $\mathrm{z}^{2} \mathrm{~s}^{2} / \mathrm{d}^{2}$ ) con nivel de confianza de $95 \%$ y costo oportunidad de $\$ 80,00$ (desviación estándar $=$ 3,3) para Medicina Familiar, para Farmacia de $\$ 55,00$ (desviación estándar $=0,67$ ) y para Laboratorio $\$ 70,00$ (desviación estándar $=2,16)$, valores estimados a partir de una prueba piloto de 10 pacientes. Valores estimados a partir de las hipótesis planteadas por los investigadores. Correspondiendo 262 adolescentes para Medina Familiar y Farmacia, y 100 para Laboratorio. El tamaño de la muestra de dividió de manera proporcional en cada una de las unida- 
des de medicina familiar incluidas. La técnica muestral fue por cuota tomando como marco muestral el listado de pacientes que acudieron al servicio de Medicina Familiar y que fueron seguidos a los servicios de Farmacia y Laboratorio. Las variables sociodemográficas para los pacientes y acompañantes incluyeron, género, edad, estado civil, ocupación y además el número de acompañantes. El costo oportunidad fue operacionalizado a través del costo minuto, se estimó para el traslado, espera y atención en los tres servicios para el paciente y sus acompañantes.

\section{Tiempo de traslado, espera y atención}

- Tiempo de traslado: promedio medido en minutos desde la sede de partida a la unidad médica multiplicado por dos para considerar el regreso.

- Tiempo de espera: promedio medido en minutos desde el momento de llegada a la unidad de medicina familiar hasta que se recibe atención en los diferentes servicios.

- Tiempo de atención: promedio medido en minutos desde el momento en que inicio hasta que finalizó la atención en cada uno de los servicios.

\section{Costo del minuto}

- Actividad laboral remunerada: se estimó preguntando directamente el salario por día, semana, quincena o mes; esta cantidad se dividió entre el total de días $(7,15,30)$ y posteriormente entre las horas laboradas al día (8), y finalmente estimada por minuto (60).

- Actividad laboral no remunerada:

- Actividades en casa: se estimó en pesos mexicanos a partir del salario del jefe de familia, dividido entre 8 horas por día y posteriormente estimada por minuto.

- Estudiante: estimado en pesos mexicanos, considerando la inversión familiar promedio anual (\$733.13) para el sostenimiento por alumno reportado por la Secretaria de Educación Pública; dividido entre el número de días escolares (210), entre el número de horas que acuden a la escuela al día (5) y posteriormente estimada por minuto ${ }^{18}$.
- Guardería: estimado en pesos; considerando la inversión del IMSS sobre el costo real día/niño para las guarderías ordinarias aplicándole el 7\% de inflación anual, dividido entre los días del año, entre las horas que labora una madre trabajadora lo que se refleja en las horas de estancia del menor en la guardería y posteriormente estimada por minuto ${ }^{19}$.

\section{Costo oportunidad}

- Costo oportunidad promedio de traslado del paciente y sus acompañantes. Calculado en base al tiempo estimado en el traslado multiplicado por el respectivo costo en minuto.

- Costo oportunidad promedio de espera del paciente y sus acompañantes. Se estimó en base al tiempo de espera para recibir atención multiplicado por el respectivo costo en minuto.

- Costo oportunidad promedio de la atención del paciente y sus acompañantes. Se estimó en base al tiempo de atención multiplicado por el respectivo costo en minuto.

- Costo oportunidad promedio por servicio. Se estimó para Medicina Familiar, Laboratorio y Farmacia, para el paciente y sus acompañantes a partir de la suma del costo oportunidad promedio en traslado, espera y atención.

$$
\underset{\mathrm{CO}=1}{\stackrel{\mathrm{k}}{\mathrm{\Sigma}} \mathrm{CO}_{\mathrm{ij}}}
$$

- Costo oportunidad promedio ponderado. Se estimó con el costo oportunidad promedio por servicio ponderado por el porcentaje de población de pacientes con o sin acompañantes.

El análisis estadístico incluyó promedios, porcentajes e intervalos de confianza del $95 \%$.

\section{Resultados}

La edad promedio de los adolescentes es de 14,26 años (IC 95\%; 13,93-14,58), mientras que en el acompañante 1 es de 38,10 años (IC $95 \% ; 36,79-39,40)$ y en el acompañante $2 \mathrm{de}$ 
12,06 años (IC 95\%; 8,99-15,13). Predomina el género femenino en los adolescentes con $64,5 \%$ (IC 95\%; 58,7-70,3) y en el acompañante 1 con $89,1 \%$ (IC 95\%; 85,3-92,9), mientras que en el acompañante 2 no existe predominio de género $50 \%$ (IC 95\%; 43,9-56,1). El 82,8\% (IC 95\%; 78,2-87,4) de los adolescentes son estudiantes, del acompañante 1 el $39,1 \%$ (IC 95\%; 33,2-45,0) es empleado y del acompañante 2 el $64,7 \%$ (IC 95\%; 58,9-70,5) es estudiante. La tabla 1 presenta la ocupación del adolescente y los acompañantes que acuden a las unidades de medicina familiar. El 89,3\% (IC 95\%; 85,6-93,0) de los adolescentes son solteros, el 63,7\% (IC 95\% 57,9$69,5)$ de los acompañantes 1 son casados y el 94,1\% (IC 95\%; 91,2-97,0) de los acompañan- tes 2 son solteros. La tabla 2 muestra el estado civil del paciente y sus acompañantes. El $75,2 \%$ (IC 95\%; 70,0-80,4) de los adolescentes acuden con un acompañante, el 19,5\% (IC $95 \% ; 14,7-24,3$ ) con dos y el 5,3\% (IC 95\%; $2,6-8,0)$ acude solo. El tiempo promedio total (traslado, espera y atención) en el servicio de Medicina Familiar es de 204,09 min (IC 95\%; 189,15-219,63), en Farmacia es de 64,17 min. (IC 95\%; 59,97-68,97) y en Laboratorio es de 91,55 min (IC 95\%; 78,54-104,56). La tabla 3 ilustra los tiempos de traslado, espera y atención para cada servicio.

Para el paciente el costo minuto promedio es de $\$ 0,0473$ (IC 95\%; 0,0353-0,0594), para el acompañante 1 de $\$ 0,3307$ (IC 95\%; 0,2983-0,3631) y para el acompañante 2 de

Tabla 1. Ocupación del adolescente y los acompañantes que acuden a las Unidades de Medicina Familiar

\begin{tabular}{|c|c|c|c|c|c|c|c|c|c|}
\hline \multirow[t]{3}{*}{ Ocupación } & \multicolumn{3}{|c|}{ Paciente } & \multicolumn{3}{|c|}{ Acompañante 1} & \multicolumn{3}{|c|}{ Acompañante 2} \\
\hline & \multirow[t]{2}{*}{ Porcentaje } & \multicolumn{2}{|c|}{ IC $95 \%$} & \multirow[t]{2}{*}{ Porcentaje } & \multicolumn{2}{|c|}{ IC 95\% } & \multirow[t]{2}{*}{ Porcentaje } & \multicolumn{2}{|c|}{ IC $95 \%$} \\
\hline & & Mín. & Máx. & & Mín. & Máx. & & Mín. & Máx. \\
\hline Estudiante & 82,8 & 78,2 & 87,4 & 1,6 & 0,1 & 3,1 & 64,7 & 58,9 & 70,5 \\
\hline Empleado & 8,0 & 4,7 & 11,3 & 39,1 & 33,2 & 45,0 & 3,9 & 1,6 & 6,2 \\
\hline Obrero & 5,0 & 2,4 & 7,6 & 7,7 & 4,5 & 10,9 & 0,0 & 0,0 & 0,0 \\
\hline Actividades en casa & 4,2 & 1,8 & 6,6 & 38,7 & 32,8 & 44,6 & 7,8 & 4,6 & 11,0 \\
\hline Profesionista & 0,0 & 0,0 & 0,0 & 6,5 & 3,5 & 9,5 & 0,0 & 0,0 & 0,0 \\
\hline Comerciante & 0,0 & 0,0 & 0,0 & 4,8 & 2,2 & 7,4 & 5,9 & 3,0 & 8,8 \\
\hline Guardería & 0,0 & 0,0 & 0,0 & 0,4 & 0 & 1,2 & 7,8 & 4,6 & 11,0 \\
\hline Casa & 0,0 & 0,0 & 0,0 & 1,2 & 0 & 2,5 & 9,8 & 6,2 & 13,14 \\
\hline
\end{tabular}

$n=262$.

Tabla 2. Estado civil del adolescente y los acompañantes que acuden a las Unidades de Medicina Familiar

\begin{tabular}{|c|c|c|c|c|c|c|c|c|c|}
\hline \multirow[t]{3}{*}{ Estado civil } & \multicolumn{3}{|c|}{ Paciente } & \multicolumn{3}{|c|}{ Acompañante 1} & \multicolumn{3}{|c|}{ Acompañante 2} \\
\hline & \multirow[t]{2}{*}{ Porcentaje } & \multicolumn{2}{|c|}{ IC $95 \%$} & \multirow[t]{2}{*}{ Porcentaje } & \multicolumn{2}{|c|}{ IC $95 \%$} & \multirow[t]{2}{*}{ Porcentaje } & \multicolumn{2}{|c|}{ IC $95 \%$} \\
\hline & & Mín. & Máx. & & Mín. & Máx. & & Mín. & Máx. \\
\hline Soltero & 89,3 & 85,6 & 93,0 & 19,0 & 14,2 & 23,8 & 94,1 & 91,2 & 97,0 \\
\hline Unión libre & 8,0 & 4,7 & 11,3 & 10,1 & 6,5 & 13,7 & 2,0 & 0,3 & 3,7 \\
\hline Casado & 2,7 & 0,7 & 4,7 & 63,7 & 57,9 & 69,5 & 3,9 & 1,6 & 6,2 \\
\hline Divorciado & 0,0 & 0,0 & 0,0 & 4,0 & 1,6 & 6,4 & 0,0 & 0,0 & 0,0 \\
\hline Viudo & 0,0 & 0,0 & 0,0 & 2,8 & 0,8 & 4,8 & 0,0 & 0,0 & 0,0 \\
\hline Separado & 0,0 & 0,0 & 0,0 & 0,4 & 0 & 1,2 & 0,0 & 0,0 & 0,0 \\
\hline
\end{tabular}

$\mathrm{n}=262$. 
\$0,0661 (IC 95\%; 0,0337-0,0986). El costo oportunidad promedio total en consulta de Medicina Familiar para el paciente es de $\$ 9,65$ (IC 95\%; 6,68-13,05) y para el acompañante 1 de $\$ 67,49$ (IC 95\%; 56,42-79,75); en Farmacia es de $\$ 3,04$ (IC $95 \% ; 2,12-4,10$ ) y de $\$ 21,22$
(IC 95\%; 17,89-25,04); y para Laboratorio es de $\$ 5,04$ (IC $95 \% ; 2,76-7,83$ ) y de $\$ 27,79$ (IC $95 \% ; 21,03-35,50)$ respectivamente. La tabla 4 muestra el costo oportunidad de traslado, espera y atención en los diferentes servicios de las Unidades de Medicina Familiar.

Tabla 3. Tiempos de traslado, espera y atención de los adolescentes y sus acompañantes en los servicios de las unidades de medicina familiar

\begin{tabular}{|c|c|c|c|c|c|c|c|c|c|c|c|c|}
\hline \multirow[t]{3}{*}{ Usuario } & \multicolumn{3}{|c|}{ Traslado } & \multicolumn{3}{|c|}{ Espera } & \multicolumn{3}{|c|}{ Atención } & \multicolumn{3}{|c|}{ Total } \\
\hline & \multirow[t]{2}{*}{ Media } & \multicolumn{2}{|c|}{ IC $95 \%$} & \multirow[t]{2}{*}{ Media } & \multicolumn{2}{|c|}{ IC $95 \%$} & \multirow[t]{2}{*}{ Media } & \multicolumn{2}{|c|}{ IC $95 \%$} & \multirow[t]{2}{*}{ Media } & \multicolumn{2}{|c|}{ IC $95 \%$} \\
\hline & & Mín. & Máx. & & Mín. & Máx. & & Mín. & Máx. & & Mín. & Máx. \\
\hline $\begin{array}{l}\text { Consulta Medicin } \\
\text { Adolescente }\end{array}$ & Familiar & & & & & & & & & & & \\
\hline $\begin{array}{l}\text { Acompañante } 1 \\
\text { Acompañante } 2\end{array}$ & 52,27 & 49,31 & 55,84 & 134,47 & 123,26 & 145,68 & 17,35 & 16,58 & 18,11 & 204,09 & 189,15 & 219,63 \\
\hline $\begin{array}{l}\text { Farmacia } \\
\text { Adolescente }\end{array}$ & & & & & & & & & & & & \\
\hline $\begin{array}{l}\text { Acompañante } 1 \\
\text { Acompañante } 2\end{array}$ & 52,27 & 49,31 & 55,84 & 9,45 & 8,46 & 10,44 & 2,45 & 2,2 & 2,69 & 64,17 & 59,97 & 68,97 \\
\hline $\begin{array}{l}\text { Laboratorio } \\
\text { Adolescente }\end{array}$ & & & & & & & & & & & & \\
\hline $\begin{array}{l}\text { Acompañante } 1 \\
\text { Acompañante } 2\end{array}$ & 54,39 & 48,74 & 60,04 & 30,07 & 23,75 & 36,39 & 7,09 & 6,05 & 8,13 & 91,55 & 78,54 & 104,56 \\
\hline
\end{tabular}

$\mathrm{n}=262$ para consulta de Medicina Familiar; $\mathrm{n}=262$ para Farmacia; $\mathrm{n}=100$ para Laboratorio.

Tabla 4. Costo oportunidad de traslado, espera, atención y total de los adolescentes y sus acompañantes y tipo de servicio de las unidades de medicina familiar

\begin{tabular}{|c|c|c|c|c|c|c|c|c|c|c|c|c|}
\hline \multirow[t]{3}{*}{ Población } & \multicolumn{3}{|c|}{ Traslado } & \multicolumn{3}{|c|}{ Espera } & \multicolumn{3}{|c|}{ Atención } & \multicolumn{3}{|c|}{ Total } \\
\hline & \multirow[t]{2}{*}{ Media } & \multicolumn{2}{|c|}{ IC 95\% } & \multirow[t]{2}{*}{ Media } & \multicolumn{2}{|c|}{ IC 95\% } & \multirow[t]{2}{*}{ Media } & \multicolumn{2}{|c|}{ IC 95\% } & \multirow[t]{2}{*}{ Media } & \multicolumn{2}{|c|}{ IC 95\% } \\
\hline & & Mín. & Máx. & & Mín. & Máx. & & Mín. & Máx. & & Mín. & Máx. \\
\hline \multicolumn{13}{|c|}{ Consulta Medicina Familiar } \\
\hline Adolescente & 2,47 & 1,74 & 3,32 & 6,36 & 4,35 & 8,65 & 0,82 & 0,59 & 1,08 & 9,65 & 6,68 & 13,05 \\
\hline Acompañante 1 & 17,29 & 14,71 & 20,28 & 44,47 & 36,77 & 52,90 & 5,74 & 4,95 & 6,58 & 67,49 & 56,42 & 79,75 \\
\hline Acompañante 2 & 3,46 & 1,66 & 5,51 & 8,89 & 4,15 & 14,36 & 1,15 & 0,56 & 1,79 & 13,49 & 6,37 & 21,66 \\
\hline \multicolumn{13}{|l|}{ Farmacia } \\
\hline Adolescente & 2,47 & 1,74 & 3,32 & 0,45 & 0,30 & 0,62 & 0,12 & 0,08 & 0,16 & 3,04 & 2,12 & 4,10 \\
\hline Acompañante 1 & 17,29 & 14,71 & 20,28 & 3,13 & 2,52 & 3,79 & 0,81 & 0,66 & 0,98 & 21,22 & 17,89 & 25,04 \\
\hline Acompañante 2 & 3,46 & 1,66 & 5,51 & 0,62 & 0,29 & 1,03 & 0,16 & 0,07 & 0,27 & 4,24 & 2,02 & 6,80 \\
\hline \multicolumn{13}{|l|}{ Laboratorio } \\
\hline Adolescente & 2,99 & 1,71 & 4,50 & 1,65 & 0,83 & 2,73 & 0,39 & 0,21 & 0,61 & 5,04 & 2,76 & 7,83 \\
\hline Acompañante 1 & 16,51 & 13,05 & 20,38 & 9,13 & 6,36 & 12,35 & 2,15 & 1,62 & 2,76 & 27,79 & 21,03 & 35,50 \\
\hline Acompañante 2 & 3,31 & 0,18 & 7,08 & 1,83 & 0,09 & 4,29 & 0,43 & 0,02 & 0,96 & 5,57 & 0,28 & 12,34 \\
\hline
\end{tabular}

La cantidad se expresa en pesos mexicanos; $n=262$ para consulta de Medicina Familiar; $n=262$ para Farmacia; $n=100$ para Laboratorio. 
ADOLESCENTES EN UNIDADES DE MEDICINA FAMILIAR

Tabla 5. Costo oportunidad promedio y ponderado por escenarios de los adolescentes y sus acompañantes

\begin{tabular}{|lcccc|}
\hline Escenarios & Adolescente & Acompañante 1 & Acompañante 2 & Ponderado \\
Medicina Familiar & 9,65 & 67,49 & 13,49 & 76,19 \\
Farmacia & 3,04 & 21,22 & 4,24 & 23,96 \\
Laboratorio & 5,04 & 27,79 & 5,57 & 32,44 \\
Medicina Familiar y Farmacia & 10,22 & 71,43 & 14,28 & 80,65 \\
\hline Medicina Familiar y Laboratorio & 11,70 & 78,77 & 15,75 & 89,37 \\
Farmacia y Laboratorio & 5,08 & 32,50 & 6,50 & 37,13 \\
Medicina Familiar, Farmacia y Laboratorio & 12,26 & 82,71 & 16,54 & 93,81 \\
\hline
\end{tabular}

La cantidad se expresa en pesos mexicanos; $n=262$ para consulta de Medicina Familiar; $n=262$ para Farmacia; $n=100$ para Laboratorio.

El costo oportunidad promedio para Medicina Familiar y Farmacia es de \$10,22 para el adolescente y de $\$ 71,43$ para el acompañante 1 , cuando acuden a los tres servicios es de $\$ 12,26$ y de $\$ 82,71$, respectivamente. El costo oportunidad promedio ponderado cuando acuden a los tres servicios es de $\$ 93,81$. La tabla 5 ilustra el costo oportunidad promedio y ponderado en cada escenario.

\section{Discusión}

Un aspecto importante de la economía de la salud es el costo oportunidad. El presente estudio analiza el costo oportunidad en los adolescentes que demandan atención en las unidades de medicina familiar y su importancia radica en que existe muy poca información al respecto, ya que los adolescentes son el grupo etario más olvidado y con la mayor subutilización de los servicios de salud. En este trabajo, se observa que el mayor porcentaje de los adolescentes acuden con al menos un acompañante, son solteros y realizan actividades no remuneradas; debido a que en el marco del costo oportunidad es necesario asignar un costo al tiempo invertido, en este trabajo la asignación contempla la inversión realizada por el sistema educativo nacional en los escolares y por el Instituto Mexicano del Seguro Social en los niños de guardería, para las actividades en casa se asigna el equivalente al salario de la pareja o jefe de familia, lo cual de alguna manera es lo más cercano a la realidad. En el análisis de los tiempos invertidos se observa que el tiempo de espera en el servicio de Medicina Familiar es mayor al de traslado y atención, lo cual repercute en el costo oportunidad. Esto es debido a que la mayor parte de los adolescentes acude a recibir atención sin cita previa. Esto representa una importante área de oportunidad para la búsqueda de estrategias dirigidas a mejorar la calidad en la atención, logrando además una menor repercusión en la economía familiar. El costo minuto promedio para el paciente es de $\$ 0,0473$, para el acompañante 1 de $\$ 0,3307$ y para el acompañante 2 de $\$ 0,0661$, esto a causa de que la mayor parte de los pacientes y acompañantes 2 son estudiantes a diferencia de los acompañantes 1 que en su mayoría son población con actividad remunerada por lo que el salario percibido por el acompañante 1 es mayor que la inversión reportada para cada estudiante y niño de guardería por el Sistema Educativo y el Instituto Mexicano del Seguro Social. Al obtener el costo oportunidad por escenarios (Medicina Familiar, Farmacia y Laboratorio), se puede observar que el servicio con un mayor costo oportunidad es sin duda el de Medicina familiar y el de Farmacia el menor costo. Sin embargo, si el paciente en la misma visita a la unidad acude al menos a dos de los diferentes servicios, el costo oportunidad se reduce. Estimar el costo oportunidad ponderado, permite observar el comportamiento de la población y de esta manera lograr una mejor planeación de los servicios de salud en el primer nivel de atención. En este estudio, se obtuvo un costo oportunidad ponderado de $\$ 76,19$ para el ser- 
vicio de Medicina Familiar, \$23,96 para farmacia y 32,44 para laboratorio. En conclusión, el costo oportunidad ponderado de los adolescentes que acuden a los 3 servicios en una sola visita es de $\$ 93,81$, correspondiendo el mayor costo oportunidad a la espera en el servicio de medicina familiar. Por lo que se sugiere implementar estrategias que disminuyan el tiempo de espera en los diferentes servicios, lo cual mejoraría notablemente la calidad de la atención que se ofrece en el instituto, pero sobre todo ocasionaría una menor repercusión en la economía de los asistentes, con lo cual se podría lograr una mayor asistencia por parte de este grupo etario, que como se ha observado en estudios previos, es el que menos acude a solicitar atención y el que sin embargo es de mayor interés para los servicios de salud, debido a que en ellos se pueden poner en marcha estrategias de prevención.

\section{Referencias}

1.- Arredondo A, Damián T, De Icaza E: Una aproximación al estudio de costos de servicios de salud en México. Salud Publica Mex 1995; 37: 437-45.

2.- Baly GA, Toledo ME, Rodríguez JF: La economía de la salud, la eficiencia y el costo de oportunidad. Rev Cubana Med Gen Integr 2001; 17(4): 395-8.

3.- Villarreal-Rios E, Mathew-Quiroz A, Garza-Elizondo ME, Núñez-Rocha G, Salinas-Martínez AM, Gallegos-

Handal M: Costo de la atención de la hipertensión arterial y su impacto en el presupuesto destinado a la salud en México. Salud Publica Mex 2002; 44: 7-13.

4.- Rodríguez-Ledesma MA, Vidal-Rodríguez C: Conceptos básicos de economía de la salud para el médico general. Rev Med Inst Mex Seguro Soc 2007; 45 (5): 523-32.

5.- Puig-Junoy J, Pinto PJL: El coste de oportunidad del tiempo remunerado en la producción de salud. Centre de Recerca en Economia i Salut Departtament d' Economia i Empresa Universitat Pompeu Fabra 2001; 1: 30.

6.- Palmer S, Raftery J: Economics notes: opportunity cost. BMJ 1999; 318: 1551-2.

7.- Arredondo A, Nájera P, Leyva R: Atención médica ambulatoria en México: el costo para los usuarios. Salud Publica Mex 1999; 41 (1): 18-26.

8.- Maldonado IG, Fragoso BJ, Orrico TS, Flores VM,
Quiroz PC, Sandoval PA: Tiempo de espera en el primer nivel para la población asegurada por el IMSS. Rev Med IMSS 2002; 40 (5): 421-9.

9.- García ALM, Villarreal RE, Vargas DRE, Martínez $G L$, Galicia RL: Costo oportunidad de las mujeres que demandan atención en la Unidad de Medicina Familiar. (Síntesis ejecutiva). Querétaro de Arteaga, Unidad de Investigación Epidemiológica y en Servicios de Salud Querétaro, IMSS; 2008.

10.- $C u F L$, Villarreal RE, Vargas DE, Galicia RL, Martínez $G L$ : Costo oportunidad en la embarazada que demanda atención en primer nivel de atención. Rev Chil Obstet Ginecol 2009; 74 (6): 345-53.

11.- Sánchez LDA, Villarreal RE, Vargas DRE, Martínez $G L$, Galicia RL: Costo oportunidad del paciente con Diabetes Mellitus tipo 2 sin Hipertensión que demanda atención en la Unidad de Medicina Familiar. (Síntesis ejecutiva). Querétaro de Arteaga, Unidad de Investigación Epidemiológica y en Servicios de Salud Querétaro, IMSS; 2009.

12.- Junco TES, Villarreal RE, Vargas DRE, Martínez GL, Galicia RL: Costo oportunidad de los niños de 0 a 9 años que demandan atención en la Unidad de Medicina Familiar. (Síntesis ejecutiva). Querétaro de Arteaga, Unidad de Investigación Epidemiológica y en Servicios de Salud Querétaro, IMSS; 2009.

13.- Méndez-López LR, Rodríguez-Romero G, Salabarria M, Izquierdo-Casino $M$ : La adolescencia y su importancia para la vida. Archivos de Medicina 2008; 4 (5): 1-7.

14.- Solís F, Mardones $G$, Castilho B: Aspectos demográficos de la población adolescente chilena. Cuad Méd Soc 1986; 27 (2): 51-62.

15.- Rivera MMT, Galicia RL, Villarreal RE, et al: Perfil de uso de los servicios de salud en primer nivel de atención por adolescentes (de 10 a 19 años). En: Villarreal RE, Martínez GL, Galicia RL.

16.- INEGI; Estadísticas. Disponible en http://www.inegi. org.

17.- Instituto Mexicano del Seguro Social: El IMSS en cifras. La salud de los adolescentes. División Técnica de información estadística en salud. Rev Med Inst Mex Seguro Soc 2008; 46 (1): 91-100.

18.- Reportes interactivos: Sistema de Información de Estadística en la Educación Básica. Disponible en http:// sieeb.basica.sep.gob.mx

19.- Guarderías: Capítulo 1. El IMSS en la Seguridad Social. Instituto Mexicano del Seguro Social (edición electrónica). 2001; Disponible en http://www.imss.gob.mx. 